know of except that now related in which an accumulation of gas in the peritoneal cavity has been survived without the closure of a wound through which the gas had passed into that cavity.

Wounds associated with emphysema of the surrounding tissues are almost always fatal unless the affected part is promptly amputated. Occasionally a cure may be effected by making very free incisions. On the other hand, the bacillus aerogenes capsulatus may circulate in the healthy tissues without harm, and the case now related shows that in exceptional circumstances recovery may follow distension of the peritoneum by gas. It seems to me that the organism gained access to the tissues during or soon after the operation, but was harmless until the pressure of the abscess diminished the vitality of the adventitious tissue between the pus and the peritoneal cavity sufficiently to allow the organism to develop in that membrane and cause gas formation, whilst some unfarourable condition in the abscess cavity prevented gas production there. Conversely the increased vitality of the tissues, brought about by the relief of tension in the abscess and in the abdominal cavity, was sufficient to arrest the aerogenic action of the bacilli.

The facts that the bacillus aerogenes capsulatus usually develops only in dead or dying tissue and the invariable association of the bacillus cedematis aerobius with septic organisms have developed a new interest since these notes were written. The men of our Expeditionary Force in France are constantly in recently-made trenches, where the opportunities are numerous for infection of wounds by anaerobic organisms found in the soil. Already a number of cases of emphysema complicating injuries have been recorded, and the bacilli under consideration have been separated from these wounds. It is obvious that to prevent the occurrence of this complication, which is almost always an indication of extreme danger, it is necessary to preserve the vitality of the parts in every possible way, and this necessity adds force to the plea made by Sir Watson Cheyne at the Medical Society and by others for an intelligent application of the teachings of Lister to the conditions of the present war. Aseptic surgery is impossible when the wounds are made septic as they are inflicted. An attempt should therefore be made to sterilise these infected wounds at the earliest possible moment by a vigorous use of antiseptics. Wimpole-street, $\mathrm{w}$.

\section{OSTEOPLASTIC RESECTION OF THE SKULL IN DECOMPRESSION OPERA- TIONS ON THE BRAIN.}

\footnotetext{
BY SPENCER MORT, M.D. GLasG., F.R.C.S. EdIN. F.R.S.E.

MEDICAL SUPRRINTEYDEXT, EDMONTON INFTRMARY, UPPER EDMONTON, $x$.
}

Two cases of brain disease with pressure on the cortex, which I have at present under treatment, have suggested a method in modification of the current procedure in decompression operations, and it may be apposite at this time. One case shows the disadvantage of removal of a large portion of bone, the other case shows the converse difficulty which may arise from replacing a temporarily resected flap of bone.

The first patient, a male, aged 40 years, was operated on some months ago by a well-known brain surgeon for intracranial pressure, probably due to tumour. The bone in the left temporal region has been removed over the silent area, apparently by Cushing's method, and at this date there is a large protrusion of the brain forming a hernia cerebri. The other patient, a female, aged 60 years, was operated on by me 14 days ago. There was a fracture of the base of the skull with intradural hæmorrhage, and pressure signs on the left side of the motor area of the cortex.

A large flap was made in the soft tissues, and four holes were drilled with a Doyen's burr at the angles of a rectangle of bone, measuring 3 inches by $2 \frac{1}{4}$ inches. With a Gigli's saw the resection was completed and the brain exposed in 15 minutes from the first incision. On incising the dura an amount of blood issued and drained away, leaving a pulseless, flat-looking area of cortex. The lower anterior drill hole was enlarged with nibbling forceps and utilised as a drain, and the bone with its cover of soft tissue was replaced. Relief from pressure was observed for a week, then evidence of irritation recommenced. In the next four days

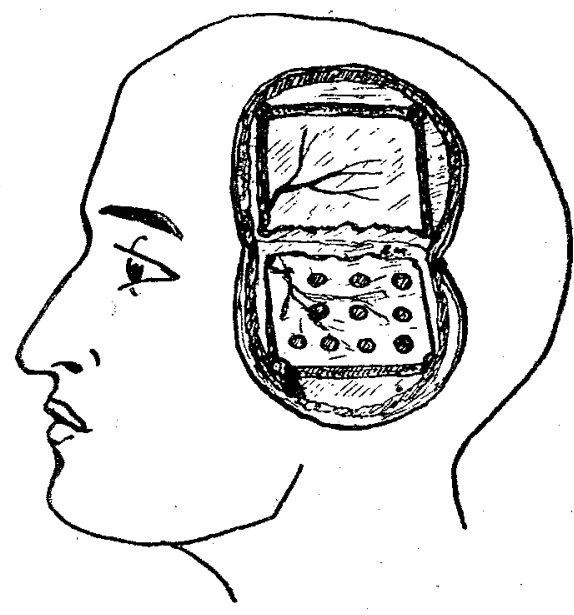

Diagrammatic sketch to illustrate " pepper-pot" trephining for the relief of intracranial pressure.

there were no less than 450 fits of Jacksonian nature counted, affecting mostly the right side of the face, with conjugate deviation of the eyes to the right and aphasia, followed by unconsciousness with Cheyne-Stokes respiration, due to pressure on the face centres and Broca's lobe. Four days after the onset of these seizures-that is, 11 days after operation-the wound was reopened and a quantity of blood-clot turned out from the brain surface, where it had collected. under the bone and caused pressure. The whole portion of resected bone was removed from the tissue flap, and the skin and muscle and aponeurosis only replaced. Immediately and obviously the pressure signs ceased, and in the next three days. there were but two slight fits observed, the breathing and pulse improved, and the general condition got better.

Now this case stands much the same as the first example quoted; there is a large bony gap, which must either remain, to the inconvenience or danger of the patient afterwards, with also the risk of hernia cerebri, or else the hiatus must be filled in by bone mosaic, osseous graft, or mechanical obturator. In similar cases, therefore, I propose to try the effect of a procedure which I have not seen described before, the object of which is to amalgamate the advantages of temporary resection with those of permanent removal of bone.

Osteoplastic reaction is eass, speedy, and gives. good access, without good drainage to prevent the 
ill-effects of any compressing agent effused beneath the window of bone. On the other hand, nibbling operations are more difficult and more prolonged, yet there is complete freedom from any fear of inefficient relief of pressure. What I suggest, then, is preliminary resection of a flap of soft tissue and bone in the usual way, following this up by "pepper pot" trephining of the flap raised. This can be done effectively and quickly with a burr, perforations $\frac{3}{8}$ to $\frac{1}{2}$ inch in diameter being drilled at close intervals from the interior of the reflected plaque, right through the osseous tissue, but not including the soft parts. The bone flap will then be a sort of retaining cage of a basket-like, fenestrated, or pepper-pot texture. This should efficiently retain the brain without risk of hernia and yet with satisfactory relief from pressure. A diagram of the idea of the operation is given.

\section{A THIRD OUTBREAK OF EPIDEMIC POLIOMYELITIS AT WEST KIRBY.}

By GEORGE JUBB, M.D. GLASG., D.P.H. OxoN., MEDICAL OFFICER OF HEALTH, HAR'TLEPOOL; LATE AC'TING MEDICAL OFFICER OF HEALTH, NORTH-WEST CHESHIRE COMBINED DISTRICTS.

THE occurrence of cases of poliomyelitis at West Kirby for the third year in succession appears to me to present so many interesting features that $I$ have ventured to put these few notes together, as so far I am not aware that there have been similar occurrences in any other district.

The first case in 1914 occurred on August 10th in a house within a quarter of a mile of the solitary case of 1912, and within the same distance of the first case of 1913, and equidistant from many of the other cases of 1913. The father of the first patient is a medical practitioner who attended two of the cases of 1913, and although this may only be a coincidence, yet it opens up a field of speculation and emphasises the importance and necessity of precautionary methods being adopted by all who come in contact with, or have to deal with, acute poliomyelitis. The following are the numbers of children attacked in each outbreak: 1912, 1 case ; 1913, 7 cases $;^{1}$ and 1914,4 cases. Some brief clinical notes of the cases may be of interest.

CaSE 1. - A male aged 3 years; West Kirby. The illness began on August 10th with violent headache but no sickness. He complained also of dryness of the mouth, and his breathing was very laboured and difficult. On the 13th twitching of his right arm and leg was noticed, and on the 15th paralysis was definitely established. The paralysis of his right arm and leg gradually disappeared, and three weeks later the boy was going about practically well. The affected muscles, however, remained flaccid, and the right knee-jerk absent. There were no other children in the house.

C.sse 2.-A female aged 6 years; Hoylake. The illness began on Sept. 22nd with headache and sickness. On the next day there was complete paralysis of the child's arms and legs. and she was unable to lift her head. Death occurred two days later (on the 25th). There were two other children in the house, one younger than this case.

CASE 3.-A male aged 3 years; Hoylake. The illness began on Oct. 1st with feverishness and vomiting, but without headache. On the 4th paralysis of the left arm was noticed. There were two other children in the house, both older than this case.

$\mathrm{C}_{A S E}$ 4.-A male aged 6 years; Hoylake. The illness began on Oct. 2nd with feverishness, headache, and

1 The Lancet, Nov. 15th, 1913, p. 1380. vomiting. On the 6th there was paralysis of both arms and legs, but left arm paralysis was pronounced. There was one younger child in the house.

\section{Distribution of Cases.}

Case 1 occurred in West Kirby in the circum. stances already mentioned. Case 2 occurred in Hoylake, about a mile away from Case 1. It may be mentioned here that Hoylake and West Kirby are practically one town and are model seaside resorts, clean, well drained, and well paved. Cases 3 and 4 were also in Hoylake, one on either side of Case 2 and about half a mile away from it. No other cases occurred in West Kirby and, as far as is known, no cases developed among visitors after leaving the district. No cases were reported from any of the adjoining districts.

\section{Possible Sources of Infection.}

Milk.-All the Hoylake cases had a common milk. supply. In outbreaks of poliomyelitis it is most unusual to find the milk-supply concerned. The apparent implication of the milk-supply here might, of course, be only another coincidence, but Case 3 was fully a mile away from Case 4, in a wellpopulated district, or rather town, and therefore the milk, with those engaged in its distribution, is bound to fall under suspicion.

Dust.--The two towns are very clean and free from ordinary dust. Sand from the shore is often blown about the towns by the wind, but this is more usual in winter than in summer.

Flies.-No excessive prevalence of flies was noticed. Against the theory of infection by means of flies it may be pointed out that poliomyelitis is increasing, while owing to the diminishing use of horses the chanees of infection by biting flies are steadily decreasing.

Other insects.-In the facts elicited regarding the cases of 1912 and 1913 there appeared to me to be some foundation for suggesting that possibly the infective agent was at times conveyed by means of lice. In this outbreak it has not been possible to collect any evidence which would substantiate this theory. On the other hand, I may say that on several occasions I have been asked if it was possible for sand fleas to convey the infection of poliomyelitis, as most of the children affected had paid recent visits to the shore.

Carriers. - It is held by many that the disease is spread by carriers, and that the cases are only "unfortunate incidents in a widespread benign infection" (R. McLaren). It is quite possible that this is so either directly or indirectly. The occurrence of these four cases would appear to support the theory of carriers, and additional support is probably given by the implication of the milk-supply.

It is worthy of note also that these four cases occurred within a very limited area, and amongst a population of 14,000 , and that the disease has taken two years to spread from West Kirby to Hoylake, a distance of only a mile, in, as already mentioned, a well-populated area.

In conclusion, I may remark that though it was not possible to trace any connexion between Case 1 in West Kirby and Case 2 in Hoylake, yet in the recurring outbreaks of three successive years it seems as if one could almost trace a case-to-case infection. I have little doubt that the many puzzles the epidemiology of poliomyelitis apparently presents will, much as in the case of malaria, be finally solved when the means of distribution of the infective agent is or are clearly established. Hartlepool. 